\title{
Cinétique de la production de matière sèche et de prélèvement d'éléments nutritifs par une cul- ture irriguée de maïs à haute potentialité de ren- dement
}

Edmond LUBET \& Christian JUSTE

I.N.R.A., Station d'Agronomie, Centre de Recherches de Bordeaux, F 33140 Pont-de-la-Maye

\begin{abstract}
Dans les conditions de milieu et pour des techniques culturales considérées comme optimales, on a suivi, grâce à 13 prélèvements de plantes, l'élaboration de la matière sèche et l'absorption des principaux éléments nutritifs par une culture de maïs à haute potentialité (variété « Roc ", indice de précocité 530).

La production totale de matière sèche atteint $21,5 \pm 1,7 \mathrm{t}$ (fig. 3) permettant de parvenir à un rendement de $115 \mathrm{q} /$ ha de grain (humidité $15 \mathrm{p} .100)$. La production maximale journalière $(482 \mathrm{~kg} / \mathrm{ha}$ ) est observée entre les stades 14 et 16 feuilles (fig. 4), 50 p. 100 de la matière sèche étant élaborée au cours du mois de juillet. Un important déficit de l'insolation (fig. 4) durant la dernière décade de juillet pourrait cependant avoir limité la production de cette période. La culture a prélevé au total dans le sol (fig. $6,8,9)$ :

- en kg/ha : $\mathrm{N}=241 ; \mathrm{P}_{2} \mathrm{O}_{5}=90 ; \mathrm{K}_{2} \mathrm{O}=322 ; \mathrm{CaO}=56 ; \mathrm{MgO}=36 ; \mathrm{S}=24 ;$

- en $\mathrm{g} / \mathrm{ha}: \mathrm{Zn}=600 ; \mathrm{Cu}=90 ; \mathrm{B}=75 ; \mathrm{Mn}=1800$.

Les plus fortes intensités d'absorption se situent entre les stades 14 et 16 feuilles (fig. 7, tabl. 3), 71 p. 100 de la potasse et 50 p. 100 des autres éléments étant prélevés en 14 jours, avec des intensités journalières de 11 et $18 \mathrm{~kg} /$ ha respectivement pour $\mathrm{N}$ et $\mathrm{K}_{2} \mathrm{O}$.

Les données obtenues devraient permettre d'affiner le raisonnement de la fumure en tenant compte des exigences des variétés très performantes dont on dispose actuellement (tabl. 4).
\end{abstract}

Mots clés additionnels : Croissance, enracinement, Zinc, Bore, Cuivre, Manganèse, Calcium, Magnésium, Phosphore, Azote, Potassium, exportations, restitutions. hybrid.

Dry matter and nutrient accumulation were measured in a high-yielding maize hybrid ('Roc', Maturity index $=530$ ) by collecting 13 successive plant samples throughout the entire growing season. The experiment was conducted under optimum environmental and cultural conditions. Dry matter accumulation reached $21.5 \pm 1.7 \mathrm{t} / \mathrm{ha}$ (fig. 3 ) with a grain yield of $11.5 \mathrm{t} / \mathrm{ha}(15 \%$ moisture). The maximum daily rate of dry matter accumulation ( $482 \mathrm{~kg} / \mathrm{ha}$ ) occurred between the 14- and 16-fully emerged leaf stages (fig. 4) and $50 \%$ of the total dry matter was accumulated in July. A strong insolation deficiency (fig. 4) observed in the last decad of July possibly decreased dry matter accumulation. The following nutrient amounts were taken up over the whole season (fig. $6,8,9):-\mathrm{kg} / \mathrm{ha}: \mathrm{N}=241 ; \mathrm{P}_{2} \mathrm{O}_{5}=90 ; \mathrm{K}_{2} \mathrm{O}=322 ; \mathrm{CaO}=56 ; \mathrm{MgO}=36$; $\mathrm{S}=24 ;-\mathrm{g} / \mathrm{ha}: \mathrm{Zn}=600 ; \mathrm{Cu}=90 ; \mathrm{B}=75 ; \mathrm{Mn}=1800$. The most rapid uptake rates were observed between the 14- and 16-fully emerged leaf stages (fig. 7, table 3);71\% of the potassium and $50 \%$ of other nutrients were accumulated in a 14-day period with daily rates of 11 and $18 \mathrm{~kg} / \mathrm{ha}$ for $\mathrm{N}$ and $\mathrm{K}_{2} \mathrm{O}$ respectively. The data presented can be used to evaluate the fertilizer requirements of present high-yielding maize hybrids (table 4).

Additional key words: Growth, rooting, Zinc, Boron, Copper, Manganese, Calcium, Magnesium, Phosphorus, Nitrogen, Potassium, exports, restitutions. 


\section{INTRODUCTION}

Le mais occupe actuellement en France 3050000 ha dont 1650000 sont destinés à la production de grain et 1400000 à l'ensilage. L'extension importante des surfaces consacrées à cette culture est à attribuer, pour une large part, à une amélioration des techniques de culture (désherbage, peuplement, fertilisation, etc...) et à la création de variétés productives, résistantes à la verse et aux maladies, bien adaptées à diverses conditions climatiques (précocité, résistance au froid...). Ces deux facteurs ont largement contribué à améliorer sensiblement les rendements et à rendre cette production attractive pour de nombreuses régions françaises : en 1950 , pour une surface totale de 325000 ha de maïs concentrée dans le Sud-Ouest, le rendement moyen était de 12,4 q/ha (CAUDERON, 1980); en 1983, il s'élevait à $63 \mathrm{q} / \mathrm{ha}$ avec une aire d'extension intéressant les $4 / 5$ du pays.

Cet accroissement spectaculaire a entraîné une augmentation sensible du niveau des intrants, et notamment de la fertilisation minérale. Pour ce qui concerne cette dernière, un des moyens pratiques permettant de la maîtriser davantage consiste à mieux connaître les potentialités de la culture (objectif de rendement) pour ajuster les apports.

Ce thème a fait l'objet d'un certain nombre de travaux (JOHNSON et al., 1966 ; HANWAY, 1962 ; HANWAY \& RUSSEL, 1969 ; COURPRON \& TAUZIN, 1971 ; BARLOY, 1983).

L'objectif de cette étude est de réactualiser l'ensemble des données relatives à la production de matière sèche du maïs, à la concentration en éléments minéraux et donc aux exportations de ces derniers dans le cas d'une variété à haute potentialité, placée dans des conditions de milieu et de culture considérées comme optimales. Grâce à de nombreuses prises d'échantillons réalisées à différentes dates, la production de matière sèche, les prélèvements maximums par la culture et le rythme d'absorption des éléments minéraux ont été appréciés à partir d'un certain nombre de plantes qui, après pesée à l'état frais et détermination de la matière sèche, ont été analysées.

\section{MATÉRIELS ET MÉTHODES}

\section{A. Localisation et description du dispositif expérimen- tal}

L'expérimentation a été implantée au printemps 1983 à proximité de Mont-de-Marsan (40), sur une parcelle de 40 ha conduite depuis 8 ans en monoculture de maïs irrigué, sans apport d'amendements organiques. Seul le grain est exporté, les résidus végétaux étant toujours restitués au sol après broyage. Les niveaux de rendement de la parcelle sont généralement élevés (90 à $100 \mathrm{q}$ de grain à 15 p. 100).

Le dispositif expérimental comporte 52 parcelles de $36 \mathrm{~m}^{2}(9 \times 4 \mathrm{~m}$, soit 5 rangs espacés de $80 \mathrm{~cm})$ qui ont permis d'effectuer 13 séries de prélèvements réparties dans le temps, ainsi qu'une parcelle de $140 \mathrm{~m}^{2}$ non fertilisée en azote, destinée à apprécier la fourniture de cet élément par le sol.

\section{B. Nature du sol (tabl. 1)}

La petite région agricole dite "Marsan " où est implanté l'essai représente en fait une zone de transition entre les sols forestiers des Landes de Gascogne (influence prépondérante du saupoudrage du sable des Landes) et le Bassin de l'Adour. Les sols qui caractérisent cette région sont des sols sableux (sables "fauves ») du Tertiaire pliocène.

La nature physique de ce milieu et sa pauvreté en matière organique sont à l'origine d'une C.E.C. et d'une R.F.U. extrêmement faibles. Les itinéraires techniques bien adaptés mais aussi le ressuyage et le réchauffement rapides de ces sols au printemps garantissent cependant la réussite des cultures d'été comme le maïs.

Pour les caractéristiques chimiques, si les apports antérieurs de $\mathrm{P}_{2} \mathrm{O}_{5}$ ont enrichi le milieu, les faibles teneurs en bases échangeables $(\mathrm{K}, \mathrm{Ca}, \mathrm{Mg})$ résultent vraisemblablement d'une possibilité de lessivage qui a été mise en évidence pour des conditions de sol et de climat très voisines (COURPRON, 1974 ; JUSTE et al., 1982).

TABLEAU 1

Caractéristiques physico-chimiques du sol (horizon $0-28 \mathrm{~cm}$ ). Soil characteristics $(0-28 \mathrm{~cm}$ depth).

\begin{tabular}{|c|c|c|}
\hline \multicolumn{3}{|c|}{ Analyse physique (\% terre fine) } \\
\hline - Argile & Inf. à & 5,1 \\
\hline - Limon fin & 2 à & 7,7 \\
\hline - Limon grossier & 20 à & 9,6 \\
\hline - Sable fin & 50 à $200 \mu$ & 17,5 \\
\hline - Sable grossier & 200 à $2000 \mu$ & 60,1 \\
\hline C.E.C., mé $/ \mathrm{kg} \ldots \ldots \ldots$. & $\ldots$ & 42 \\
\hline Densité apparente $\ldots \ldots \ldots$ & & 1,58 \\
\hline Carbone organique (Anne). & $\ldots \%$ & 6,0 \\
\hline Matière organique ....... & $\ldots \%$ & 10,8 \\
\hline Azote (Kjeldahl) . . . . . . & $\ldots \%$ & 0,56 \\
\hline Rapport $\mathrm{C} / \mathrm{N} \ldots \ldots \ldots$ & $\ldots \ldots$ & 10,7 \\
\hline pH-eau $\ldots \ldots \ldots \ldots \ldots$ & & 5,1 \\
\hline $\mathrm{pH}-\mathrm{KCl} \mathrm{N} \ldots \ldots \ldots \ldots$ & & 4,6 \\
\hline Acide phosphorique (Dyer) & .. \% & 0,448 \\
\hline Calcium échangeable ...... & .. \% & 0,225 \\
\hline Magnésium échangeable ... & $\ldots \ldots \% \%$ & 0,028 \\
\hline Potassium échangeable .... & $\ldots \ldots \% \%$ & 0,051 \\
\hline
\end{tabular}

\section{Conduite de l'essai}

Cette étude a été réalisée dans des conditions de culture tout à fait identiques à celles pratiquées par les maïsiculteurs (utilisation de tracteur, outils de préparation du sol, semoir, irrigation au canon enrouleur). Seules, les fertilisations minérales ont été apportées manuellement.

\section{Fertilisation minérale}

L'ensemble du dispositif a reçu la fertilisation suivante (en $\mathrm{kg} / \mathrm{ha}$ d'unités fertilisantes) appliquée avant et après labour :

\footnotetext{
$-\mathrm{P}_{2} \mathrm{O}_{5}: 235$ (scories et superphosphate à 45 p. 100) ;

$-\mathrm{K}_{2} \mathrm{O}: 225$ (chlorure de potassium);

- $\mathrm{CaO}: 1000$ (carbonate de calcium et scories) ;

- MgO : 400 (carbonate magnésien) ;

- $\mathrm{Zn}$ et $\mathrm{Cu}: 12$ et 6 respectivement (complexe solide d'oligo-éléments).
} 
La fertilisation azotée a été fractionnée en 2 apports effectués en cours de végétation sous forme de nitrate d'ammoniaque :

- 80 unités, en plein, au stade 3 feuilles du maïs,

- 220 unités, en localisation dans l'interligne et enfoui par un binage, au stade 10 feuilles.

Ces apports, qui peuvent paraître excessifs pour certains éléments, se justifient par le souci de se mettre à l'abri de tout facteur limitant.

\section{Modalités de culture}

Labour à $25 \mathrm{~cm}$ (fin avril) ; semis début mai (interligne à $80 \mathrm{~cm}$, distance entre rangs sur la ligne : 16,7 cm) de la variété «Roc », hybride simple, denté, tardif (indice de précocité : 530) ; 5 irrigations correspondant à un total de $149 \mathrm{~mm}$ ont permis de couvrir le déficit hydrique estival.

\section{Modalités de prélèvement, d'échantillonnage des végétaux et déterminations réalisées}

Les différents stades dont il est fait état au cours de ce travail ne représentent pas des stades physiologiques bien définis mais les stades végétatifs (exemple : nombre de feuilles) auxquels se trouvaient les plantes au moment du prélèvement.

\section{Prélèvement et échantillonnage des parties aérien- nes (tabl. 2)}

Pour chaque prélèvement, 7 plantes ont été récoltées sur une longueur de $1,20 \mathrm{~m}$ représentant une placette, et cela, pour chacune des 4 parcelles élémentaires volontairement choisies non contiguës.

La dimension modeste du prélèvement (moins de $1 \mathrm{~m}^{2}$ sur $36 \mathrm{~m}^{2}$ ) élimine l'effet interparcellaire de type « fenêtre » pouvant influencer ultérieurement le développement des plantes voisines.

Après la collecte, les 28 plantes des 4 lots ont été pesées individuellement, en prenant en compte :

- la totalité de la plante jusqu'au $8^{\mathrm{e}}$ prélèvement ;

- les parties aériennes « non épi » et épi (spathes, rafles et grains) pesées séparément, du $9^{\mathrm{e}}$ au $11^{\mathrm{e}}$ prélèvement.

TABLEAU 2

Calendrier des prélèvements.

Sampling dates.

\begin{tabular}{rcccc}
\hline $\begin{array}{c}N^{\circ} \text { du } \\
\text { prélè- } \\
\text { vement }\end{array}$ & $\begin{array}{c}\text { Date du } \\
\text { prélè- } \\
\text { vement }\end{array}$ & $\begin{array}{c}\text { Pas de } \\
\text { temps } \\
\text { (jours) }\end{array}$ & $\begin{array}{c}\text { Age du } \\
\text { maïs } \\
\text { (jours) }\end{array}$ & Stade du maïs \\
\hline 1 & 11.06 & 7 & 27 & 8 feuilles \\
2 & 18.06 & 7 & 34 & $9-10$ feuilles \\
3 & 25.06 & 7 & 41 & $11-12$ feuilles \\
4 & 02.07 & 7 & 48 & 13 feuilles \\
5 & 09.07 & 7 & 55 & 14 feuilles \\
6 & 16.07 & 7 & 69 & $15-16$ feuilles \\
7 & 23.07 & 7 & 69 & Panicule cornet \\
8 & 30.07 & 7 & 76 & Sortie soies \\
9 & 06.08 & 7 & 83 & Gonflement épi \\
10 & 13.08 & 14 & 90 & Grain laiteux \\
11 & 27.08 & 16 & 104 & Grain pâteux \\
12 & 12.09 & 19 & 120 & Grain pâteux dur \\
13 & 01.10 & & 139 & Récolte : grain 29\% \\
\hline \hline
\end{tabular}

Une différenciation plus complète a été réalisée pour les $12^{\mathrm{e}}$ et $13^{\mathrm{e}}$ prélèvements en séparant : tiges, feuilles avec gaines, spathes et pédoncules, rafles, grains. Après le $5^{\text {e }}$ prélèvement et pour des raisons d'encombrement, 3 plantes seulement ont été conservées mais en tenant compte cependant du poids moyen frais des 7 plantes toujours prélevées et pesées une à une. Les 4 lots issus de chaque parcelle ont été rincés à l'eau distillée, séchés à l'étuve ventilée $\left(105^{\circ} \mathrm{C}\right)$, puis pesés et broyés.

\section{Prélèvement et échantillonnage du système racinaire} (fig. 1)

Les observations et mesures ont été réalisées du 9 au 11 août (12 à $14 \mathrm{j}$ après la floraison), c'est-à-dire, à un stade de développement de la plante à partir duquel, selon certains auteurs (MENGEL \& BARBER, 1974 ; DERIEUX, 1983), la croissance du système racinaire a atteint son maximum.

La caractérisation de l'enracinement a été réalisée en assimilant une plante à un échantillon élémentaire ; la distance entre plantes étant de $16,7 \mathrm{~cm}$ et l'interligne de $80 \mathrm{~cm}$, le site surfacique exploré a été ramené à ces dimensions, la plante choisie se trouvant exactement au centre du rectangle ainsi défini.

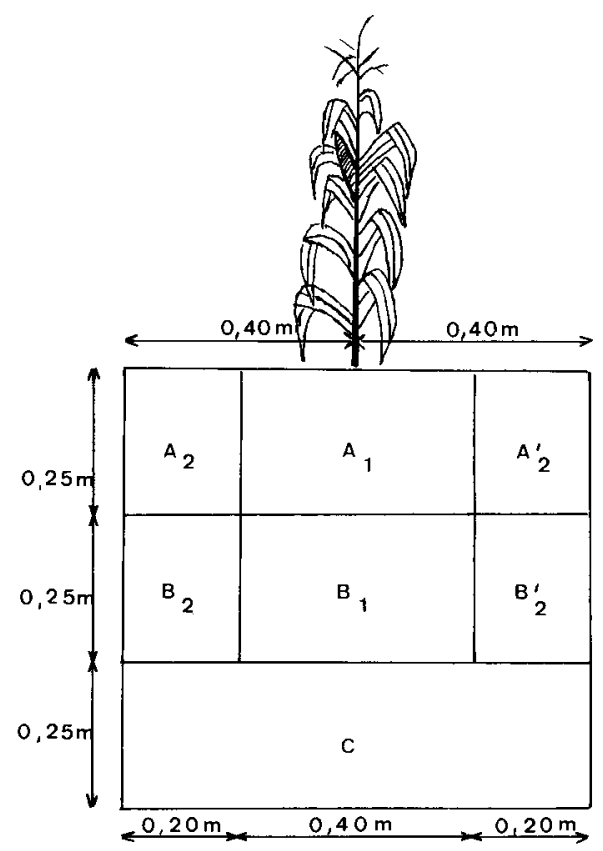

\begin{tabular}{|c|c|c|c|c|}
\hline $\begin{array}{l}\text { Horizon } \\
\text { prélevé }\end{array}$ & $\begin{array}{c}\text { Poids } \\
\text { Mat. sèche } \\
\text { des racines } \\
(\mathrm{g})\end{array}$ & $\%$ total & $\begin{array}{l}\text { Interv. de } \\
\text { confiance } \\
(5 \%)\end{array}$ & $\begin{array}{c}\text { C.V. } \\
0 \%\end{array}$ \\
\hline $\mathbf{A}_{1}$ & 26,01 & 76,5 & 5,94 & 9,2 \\
\hline $\mathrm{A}_{2}+\mathrm{A}_{2}^{\prime}$ & 3,60 & 10,6 & 1,94 & 21,7 \\
\hline $\mathbf{B}_{1}$ & 2,44 & 7,2 & 0,79 & 13,0 \\
\hline $\mathrm{B}_{2}+\mathrm{B}_{2}^{\prime}$ & 1,68 & 5,0 & 0,57 & 13,6 \\
\hline $\mathrm{C}^{2}$ & 0,26 & 0,8 & 0,39 & 61,7 \\
\hline$\Sigma$ & 33,99 & & 5,18 & 6,1 \\
\hline
\end{tabular}

Figure 1

Schéma de prélèvement et répàrtition du système racinaire au stade brunissement des soies ( $g$ de matière sèche de racines par plante); Maize root sampling system and root distribution at the silking stage ( $g$ dry matter per plant). 
Pour chaque prélèvement, après creusement d'une fosse de dégagement de 1,20 $\mathrm{m}$ de profondeur, on a découpé, avec un couteau à longue lame et selon un profil rectiligne perpendiculaire à la ligne de semis, 7 cubes dont la face frontale est représentée figure 1 . Ces cubes ont été recueillis dans un bac plaqué contre la paroi du profil (pour le sol considéré, cette opération de découpage de blocs est facilitée lorsque le matériau est à 20 p. 100 d'humidité).

Immédiatement après le prélèvement, les racines ont été séparées du sol en soumettant ce dernier à 3 brassages successifs dans une bétonnière (PIERRE, comm. pers.). Après décantation de quelques minutes, la terre se dépose alors que les débris végétaux - y compris les racines - remontent à la surface. On sépare celles-ci par tamisage (maille $n^{\circ} 8$ ) et on renouvelle ce traitement jusqu'à ce qu'il n'y ait plus de racines en flottation.

La présence de débris végétaux anciens nécessite un triage à la pince brucelle, faisant intervenir les différences de couleur entre ces résidus et les racines vivantes de maïs. L'absence sur le terrain de toute adventice annuelle ou vivace garantit l'appartenance au maïs de toute racine vivante ainsi isolée.

La M.S.R. (matière sèche racinaire) qui correspond aux autres dates de prélèvement des parties aériennes a été calculée par interpolation, en tenant compte des valeurs obtenues pour 6 prélèvements élémentaires et des données fournies par les travaux de GIRARDIN (1982).

Cette méthode est bien adaptée à la texture sableuse non caillouteuse du sol du dispositif. L'étude statistique de la variabilité des résultats fournis par les 6 prélèvements en confirme la validité.

\section{Déterminations réalisées}

Outre la détermination du poids des échantillons récoltés, on a effectué le dosage de $\mathrm{N}$ (méthode Kjeldahl) et de $\mathrm{P}, \mathrm{K}, \mathrm{Ca}, \mathrm{Mg}, \mathrm{S}, \mathrm{B}, \mathrm{Zn}, \mathrm{Cu}$ et $\mathrm{Mn}$ par calcination des poudres et reprise des cendres par l'acide chlorhydrique $5 \mathrm{~N}$. Le dosage des métaux a été réalisé par spectrophotométrie d'absorption atomique et celui de $\mathrm{P}$ par colorimétrie du complexe phosphomolybdique réduit (Laboratoire d'Analyses végétales de l'I.N.R.A., Bordeaux).

\section{RÉSULTATS ET DISCUSSION}

\section{A. Caractérisation des conditions climatiques}

Particulièrement importantes pour la croissance et le développement du maïs, les conditions climatiques qui ont régné durant la phase expérimentale sont résumées dans la figure 2 .

\section{B. Production de matière sèche}

\section{Matière sèche aérienne (M.S.A.)}

Les résultats concernant le rythme de production de la M.S.A. sont représentés par la figure 3 ; sur cette dernière, on a fait apparaître, outre la courbe moyenne, les 2 courbes enveloppes des intervalles de confiance calculés pour un seuil de signification de 5 p. 100. Le rythme de formation est conforme à celui observé par d'autres auteurs (HANWAY, 1962, 1969 ;

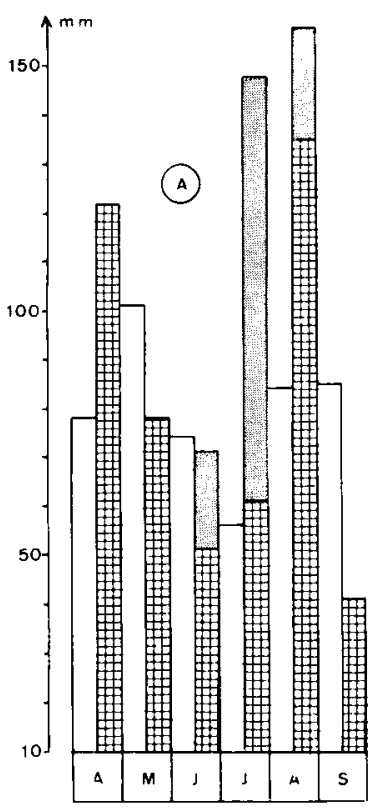

Figure 2

Eléments de climatologie (1983 et moyenne sur 12 ou 30 ans); Climatological data (1983 and average for a 12-or 30 year period).

A) Pluviométrie ; Rainfall

- Moyenne 30 ans (Mont-de-Marsan); Average values for a 30 year period (Mont-de-Marsan)

曲 1983 (Site expérimental) ; 1983 (Experimental area)

- Irrigation 1983
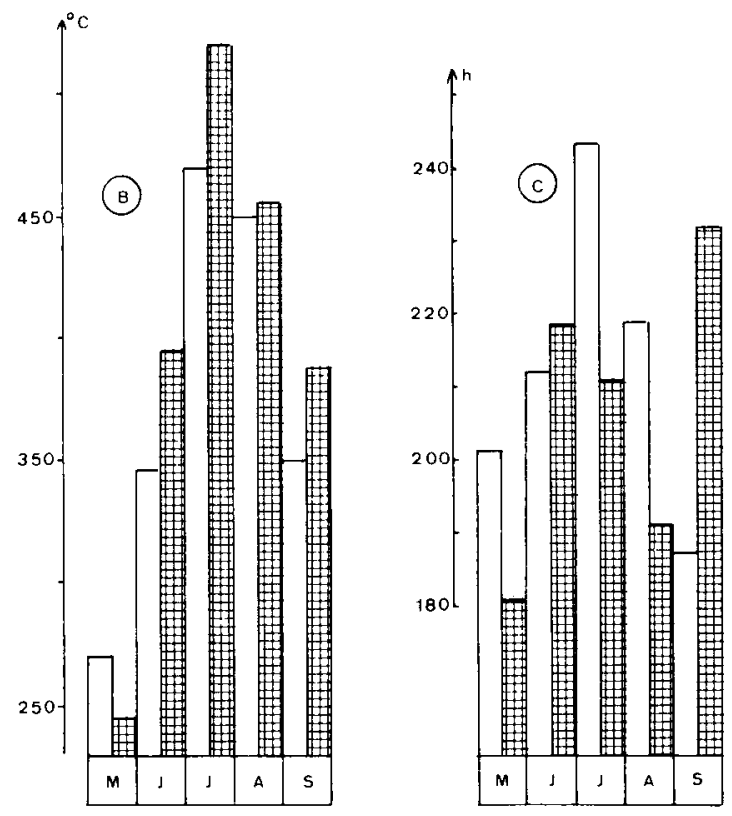

B) Somme des températures mensuelles (base $6{ }^{\circ} \mathrm{C}$ ); Sum of monthly iemperatures above $\left.6{ }^{\circ} \mathrm{C}\right)$

- Moyenne 12 ans (Mont-de-Marsan); Average values for a 12 year period (Mont-de-Marsan)

囲聿 1983 (Mont-de-Marsan)

C) Durée d'insolation mensuelle; Monthly insolation time

$\square$ - Moyenne 30 ans (Mont-de-Marsan); Average values for a 30 year period (Mont-de-Marsan)

- 1983 (Mont-de-Marsan) 
COURPRON \& TAUZIN, 1971 ; MENGEL \& BARBER, 1974 ; BARLOY, 1983), mais on relève cependant des différences importantes en ce qui concerne les niveaux et l'intensité de la production :

a) Niveau de production - L'obtention d'une quantité de M.S.A. qui peut atteindre 20,9 t/ha est remarquable mais non surprenante dans le Sud-Ouest atlantique, où les niveaux de production de l'ordre du rendement moyen obtenu sur l'essai (115 q de grain à 15 p. 100) sont fréquemment atteints.

b) Intensité de la production journalière - Elle est calculée à partir de la différence de production de M.S. obtenue entre 2 prélèvements ; la figure 4 fait apparaître que cette intensité, qui atteint son maximum durant

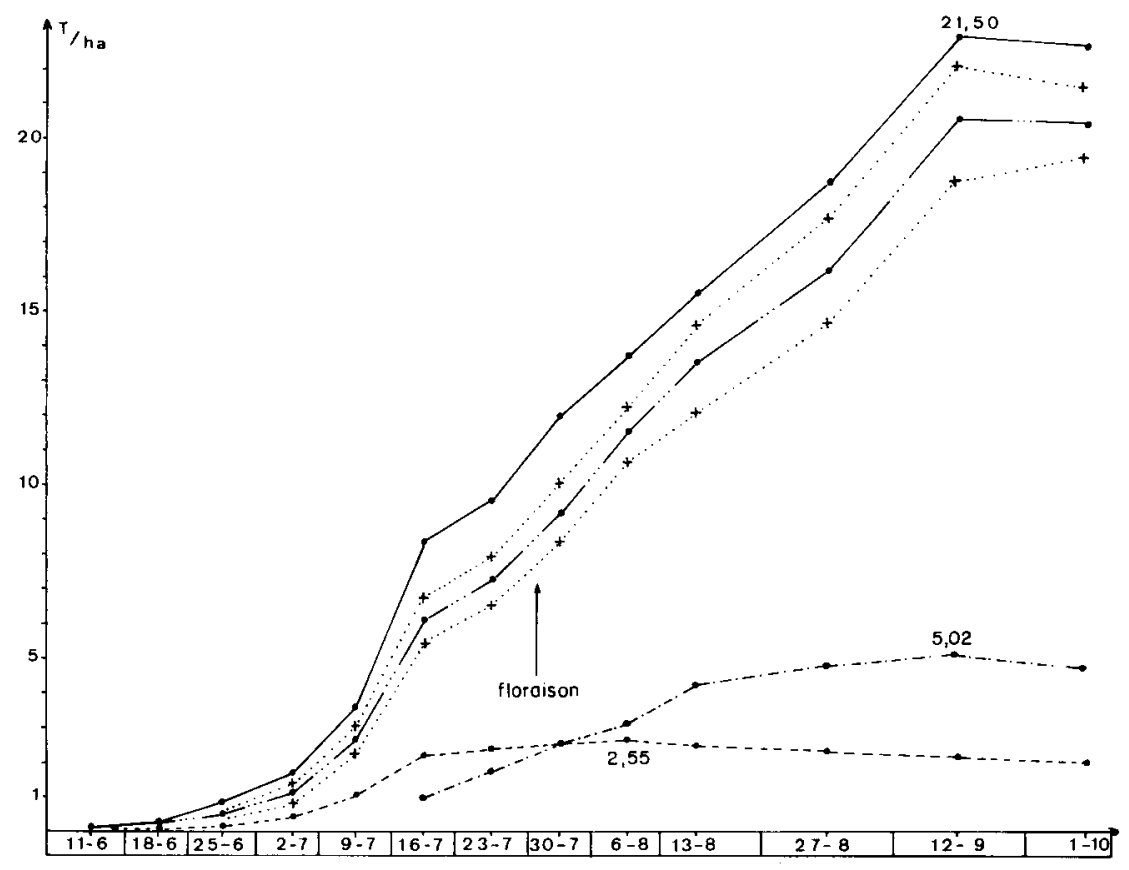

Figure 3

Rythme de production de la matière sèche du mais $(t / h a)$; Dry matter accumulation in maize plant $(t / h a)$.

- - - Matière sèche totale; Total dry matter

-.... - Matière sèche parties aériennes (courbe moyenne); Dry matter of above-ground plant parts (average curve)
+........ - Courbes enveloppes des intervalles de confiance au seuil de 5 p. 100 (parties aériennes); Confidence limits for $P=0.05$ (above-ground plant parts).

- Matière sèche système racinaire; Dry matter of roots

- Matière sèche parties aériennes (parcelle sans azote) ; Dry matter of above-ground plant parts (plot without nitrogen).

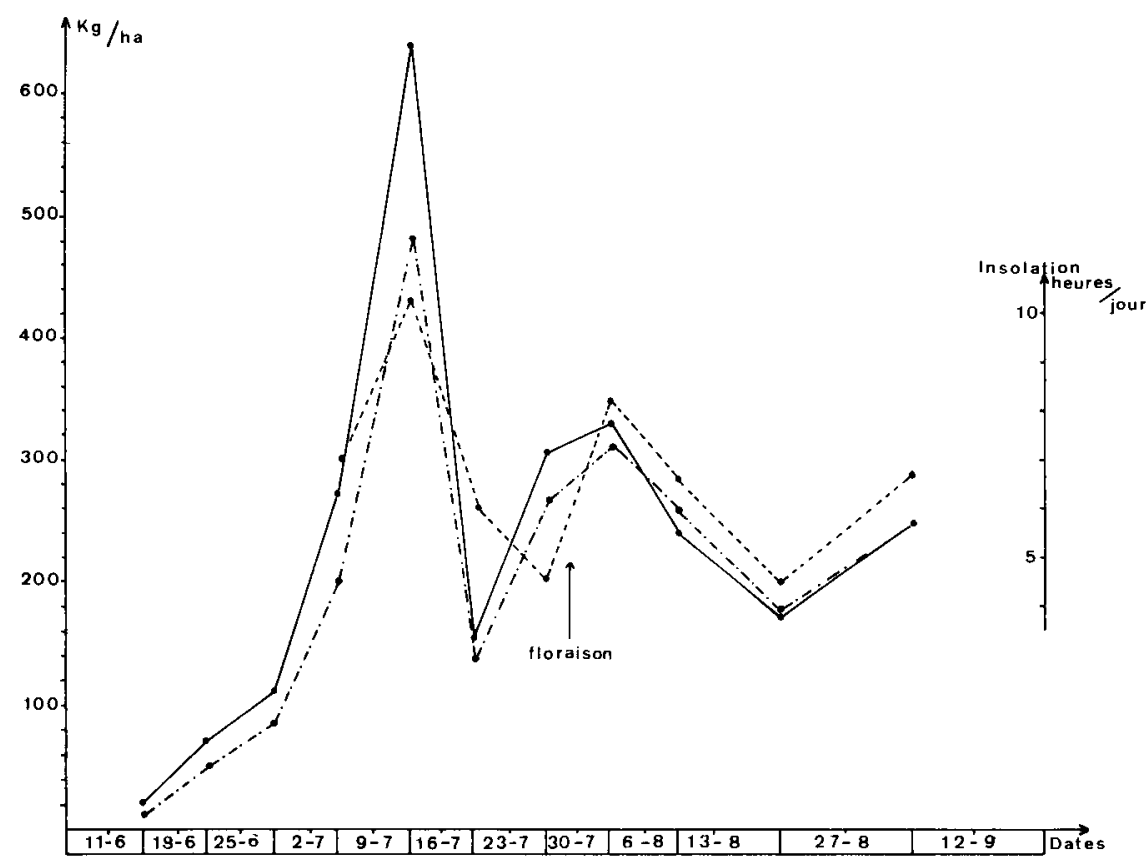

Figure 4

Production journalière de matière sèche ( $\mathrm{kg} / \mathrm{ha} / \mathrm{jour})$ et insolation moyenne (heures/jour); Daily rate of dry matter accumulation in maize plant ( $\mathrm{kg} / \mathrm{ha} /$ day) and insolation time ( $\mathrm{h} /$ day).
- Matière sèche totale; Total dry matter

- Matière sèche parties aériennes; Above-ground plant parts dry matter

- Insolation moyenne; Insolation time. 
le mois de juillet (50 p. 100 de la M.S. totale produits au cours de ce mois), présente cependant des fluctuations notables au cours du temps. Ainsi, entre le $5^{\mathrm{e}}$ et le $6^{\mathrm{e}}$ prélèvement, cette production s'est élevée à $482 \mathrm{~kg} / \mathrm{ha}$ de M.S. Cette vitesse de croissance considérable est à rapprocher des valeurs citées par MOROTGAUDRY \& JOLIVET (1983) qui signalent des productions journalières de cet ordre de grandeur en Californie, productions correspondant à l'utilisation de 7 à 9 p. 100 de l'énergie lumineuse reçue. Cependant, pour l'intervalle suivant, l'intensité s'effondre $(141 \mathrm{~kg} / \mathrm{ha} / \mathrm{j})$, puis se relève sensiblement ensuite $(266 \mathrm{~kg} / \mathrm{ha} / \mathrm{j})$. On peut se demander si ces fluctuations, non explicables par la valeur élevée des sommes de température, ne sont pas à mettre en parallèle avec les durées d'insolation qui ont présenté durant cette période des variations sensibles (fig. 4) en s'écartant notablement des moyennes décadaires. L'absence de toute donnée concernant le rayonnement global dans le Sud du département des Landes n'a pas permis de mieux étayer cette hypothèse.

\section{Matière sèche racinaire (M.S.R.)}

Lors de l'examen du système racinaire, les plantes (stade brunissement des soies) ont une hauteur moyenne (du sol à l'extrémité supérieure de la panicule) de $3,37 \mathrm{~m}$ et un diamètre moyen de tige (entre les 2 premiers entre-nœuds de la base) de 23,7 mm.

a) Niveau de production (fig. 3) : La production maximale de M.S.R. (2,55 t/ha) représente 19 p. 100 de la matière sèche totale ou 23,5 p. 100 de la M.S.A. Les valeurs habituellement citées varient sensiblement selon les auteurs (MENGEL \& BARBER, 1974 ; GIRARDIN, 1982 ; BARLOY, 1983 ; DERIEUX, 1983), variations qui peuvent s'expliquer par la variété et le peuplement, la méthodologie du prélèvement, les conditions du milieu et notamment le stress hydrique qui peut jouer sur le rapport racines/parties aériennes (BROUWER, 1963 ; SHARP \& DAVIES, 1979). b) Répartition spatiale (fig. 1) : Elle se caractérise par:

- une concentration importante du système racinaire dans la couche labourée ( 87 p. 100 de la M.S.R.) ;

- une colonisation moindre de l'horizon sous-jacent $25-50 \mathrm{~cm}$ (12 p. 100 de la M.S.R.);

- la faible exploration de l'horizon $50-70 \mathrm{~cm}$ (0,8 p. 100 de la M.S.R.) ;

- l'absence de toute racine au-delà de l'horizon $75 \mathrm{~cm}$.

L'examen du profil n'ayant pas révélé de zones indurées ou réductrices, cette faible exploration des couches profondes du sol peut s'expliquer par l'influence de la fertilisation appliquée majoritairement en surface et, surtout, par une alimentation hydrique ajustée aux besoins du maïs dont le profil racinaire est très sensible aux conditions d'alimentation en eau (BONHOMME, 1983).

\section{Matière sèche totale (M.S.T.) (fïg. 3)}

Le maximum de M.S.T. (21 $479 \mathrm{~kg} \pm 1$ 700) correspond à l'exploitation en ensilage. Les teneurs en matière sèche sont alors respectivement de 52 p. 100 pour le grain (pâteux dur), de 20 p. 100 pour les tiges et les feuilles, de 50,6 p. 100 pour la totalité de l'épi et de 28,7 p. 100 pour la plante entière. Entre ce dernier stade et la récolte (grain à 29 p. 100 d'humidité ; poids maximum de 1000 grains à 0 p. 100 d'humidité $=288 \mathrm{~g}$ ), la variation pondérale de matière sèche des parties aériennes végétatives (principalement des tiges) est négative et correspond sensiblement à la variation positive de la matière sèche du grain (fig. 5).

\section{Prélèvement d'éléments nutritifs}

Les prélèvements cumulés en éléments majeurs et méso-éléments ainsi que leur intensité d'absorption

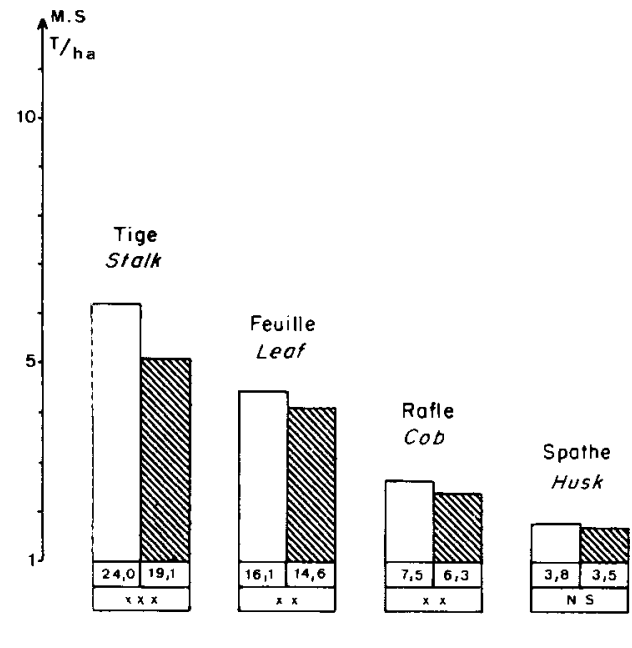

Figure 5

Répartition de la matière sèche entre les différents organes au stade ensilage ( $12^{e}$ prélèvement) et à maturité complète ( $13^{e}$ prélèvement) ;

Dry matter distribution between the different plant parts at ensilage and complete maturity stages.

\footnotetext{
$\square$ - Stade ensilage ; Ensilage stage

- Stade maturité complète ; Complete maturity stage

- Différence significative au seuil de 1 p. 1000 ; Signifi-

$\times \times \times \quad$ Différence significative au seuil de
cant at 0.001 level of probability

$\times \times \quad$ Différence significative au seuil de 1 p. 100 ; Significant at 0.01 level of probability.
} 
mesurée au cours du temps sont représentés respectivement sur les figures 6 et 7 .

Deux paramètres permettent de mieux caractériser les demandes instantanées :

a) Intensité des prélèvements journaliers (fig. 7) : Pour la plupart des éléments, l'intensité des prélèvements journaliers la plus forte se situe à la période où l'intensité de la production de matière sèche est ellemême à son maximum. Dans les conditions de cette expérimentation, cette période se situe dans les $40 \mathrm{j}$ qui précèdent la floraison, c'est-à-dire, entre les stades 12 à 16 feuilles. Les prélèvements journaliers atteignent alors, sur une durée de $7 \mathrm{j}, 11,4 \mathrm{~kg} / \mathrm{ha}$ pour $\mathrm{N}$ et $18,3 \mathrm{~kg} /$ ha pour $\mathrm{K}_{2} \mathrm{O}$.

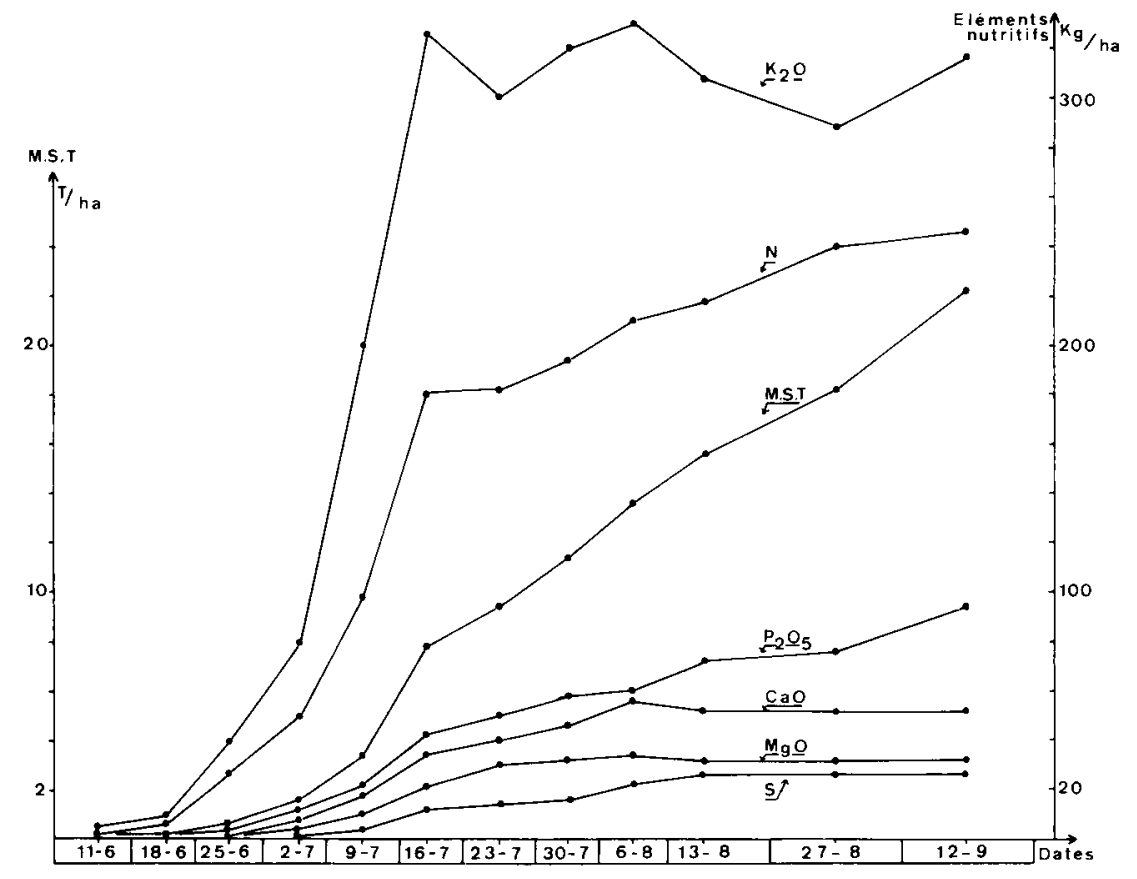

Figure 6

Rythme de production de la matière sèche totale $(t / h a)$ et du prélèvement des principaux éléments nutritifs par l'ensemble de la plante

$(\mathrm{kg} / \mathrm{ha})$; Dry matter accumulation (t/ha) and nutrient uptake by the whole maize plant $(\mathrm{kg} / \mathrm{ha})$.

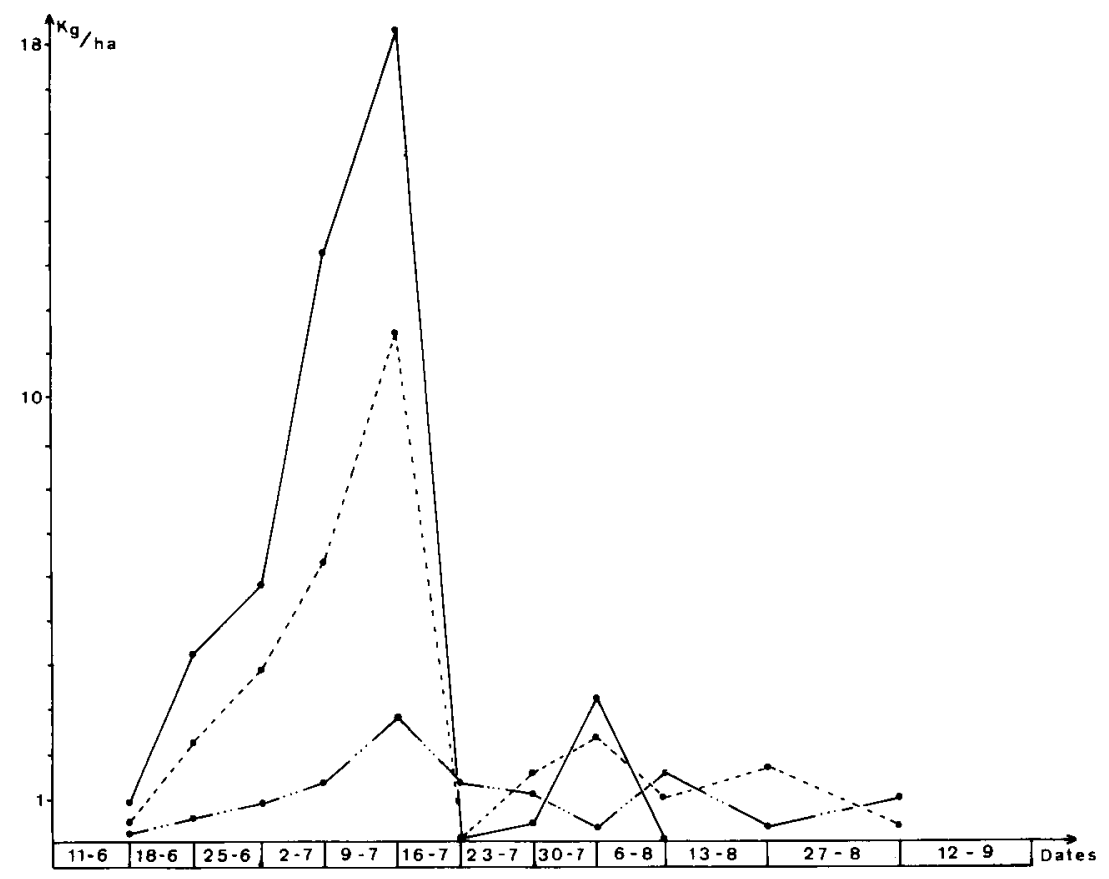

Figure 7

Prélèvement journalier des principaux éléments nutritifs; Daily rate of nutrient uptake by maize plants.

$$
\mathrm{K}_{2} \mathrm{O} ;-\cdots-\cdots+\cdots \rightarrow \mathrm{P}_{2} \mathrm{O}_{5}
$$


b) Période de prélèvement maximum (tabl. 3) : Durant la phase de développement la plus active du cycle, la hauteur moyenne des plantes passe de 102 à $264 \mathrm{~cm}$ en $14 \mathrm{j}$. Si, pendant cette courte période, 30 p. 100 de la M.S.T. a été élaborée, les prélèvements d'éléments représentent des pourcentages de l'absorption totale très supérieurs ; il n'y a donc pas systématiquement parallélisme entre formation de M.S. et prélèvement d'éléments nutritifs.

\section{Azote}

a) Besoins globaux : La courbe moyenne des prélèvements et les 2 courbes enveloppes des intervalles de confiance au seuil de 5 p. 100 sont représentées dans la figure 8. La grande variabilité des quantités prélevées

TABLEAU 3

Production de matière sèche et prélèvement en éléments nutritifs du stade 13 feuilles au stade 16 feuilles (14 jours) (en p. 100 du total)

Dry matter accumulation and nutrient uptake between the 13- and 16-fully emerged leaf stages (14 days).

\begin{tabular}{|c|c|}
\hline Matière sèche totale & 29,6 \\
\hline $\mathbf{N} \ldots \ldots \ldots \ldots$ & 52 \\
\hline 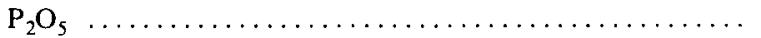 & 32 \\
\hline 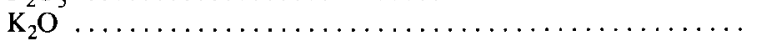 & 71 \\
\hline 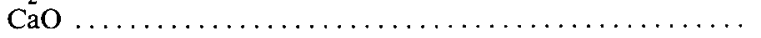 & 50 \\
\hline $\mathrm{MgO} \ldots \ldots \ldots \ldots \ldots \ldots \ldots \ldots \ldots \ldots \ldots \ldots$ & 51 \\
\hline 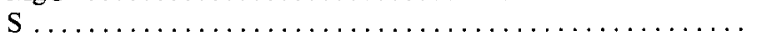 & 37 \\
\hline 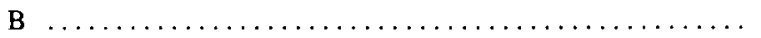 & 39 \\
\hline$\ldots \ldots \ldots \ldots \ldots \ldots \ldots \ldots \ldots \ldots$ & 52 \\
\hline$\ldots \ldots \ldots \ldots \ldots \ldots \ldots \ldots \ldots \ldots$ & 53 \\
\hline$\ldots \ldots \ldots \ldots$ & 50 \\
\hline
\end{tabular}

est évidente. Pour une grande part, elle est à attribuer aux concentrations relevées dans les différents organes issus des $12^{\mathrm{e}}$ et $13^{\mathrm{e}}$ prélèvements. Les résultats font apparaître en effet une fluctuation importante de la teneur en $\mathrm{N}$ des tiges (C.V. de l'ordre de 40 p. 100) alors que les valeurs observées pour les autres organes apparaissent beaucoup plus régulières (C.V. inférieurs à 6 p. 100). On peut penser, compte tenu de la prépondérance de la tige dans la constitution de la biomasse, que cet organe est à l'origine de la variabilité élevée de la concentration moyenne en $\mathrm{N}$ tout au long du cycle. Si l'on se réfère à la courbe moyenne (fig. 6), on constate que, pour produire $21,5 \mathrm{t} / \mathrm{ha}$ de M.S., le maïs a prélevé $241 \mathrm{~kg}$ de $\mathrm{N}$ dont on retrouve $36 \mathrm{~kg}$ dans le système racinaire et $109 \mathrm{~kg}$ dans le grain. La richesse en $\mathrm{N}$ de ce dernier $(1,35 \mathrm{p}$. 100 , soit $1,15 \mathrm{~kg} \mathrm{~N} / \mathrm{q}$ de grain à 15 p. 100 d'humidité) apparaît, dans ce cas précis, plus faible que celle habituellement constatée. De nombreuses observations réalisées par ailleurs n'autorisent cependant pas à relier systématiquement teneur en azote du grain et niveau de rendement.

b) Rythme d'absorption: Schématiquement, le rythme d'absorption de $\mathrm{N}$ présente 3 phases d'intensité différente :

- faible jusqu'au stade 11-12 feuilles (10 p. $100 \mathrm{du}$ total absorbé),

- très forte du stade 12 feuilles au brunissement des soies ( 75 p. 100 du total absorbé),

- modéré du gonflement de l'épi au stade ensilage (15 p. 100 du total absorbé).

c) Contribution du sol: La contribution du sol à la fourniture de $\mathrm{N}$ (essentielle pour le raisonnement de la fertilisation azotée) s'est avérée médiocre, pour les conditions pédoclimatiques de cette expérimentation ; en effet, l'exportation totale de $\mathrm{N}$ par les plantes issues

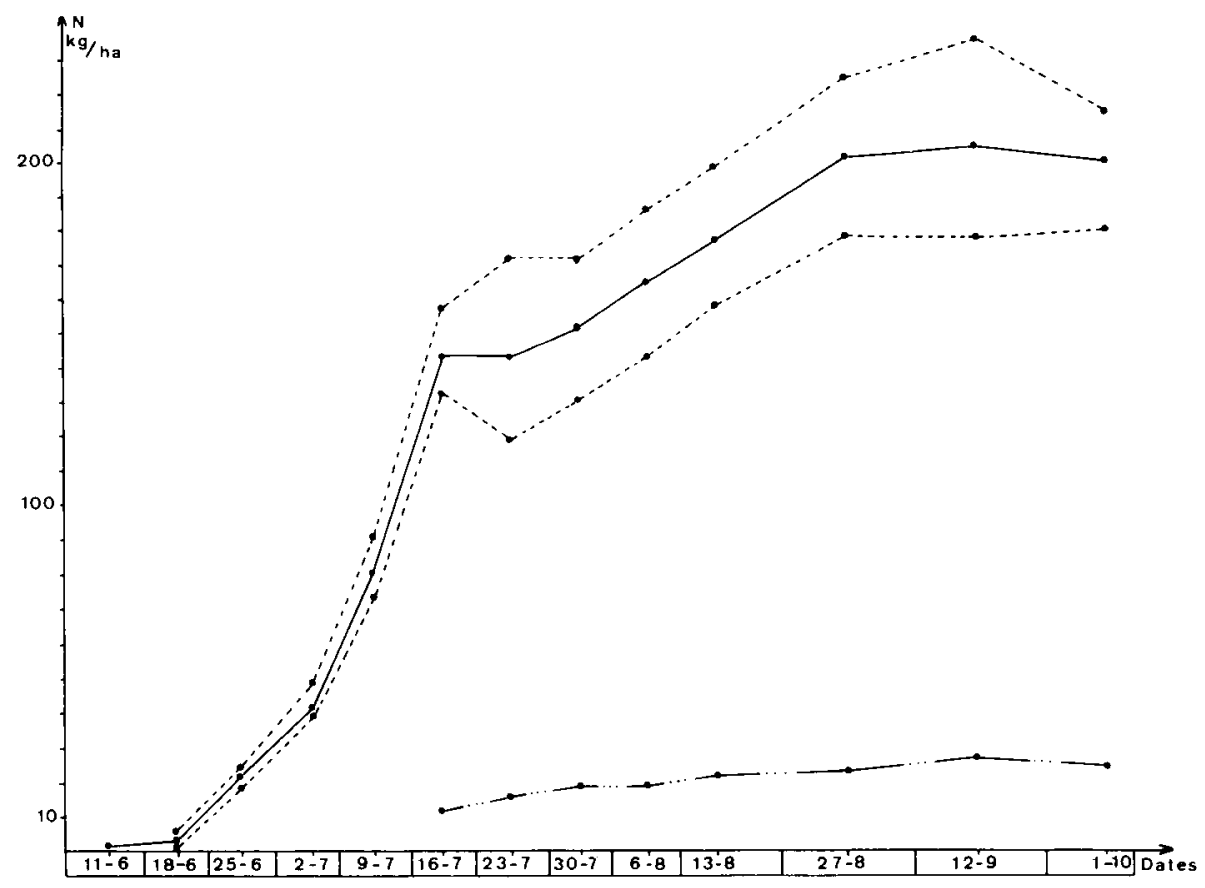

Figure 8

Rythme d'accumulation de l'azote dans les parties aériennes ; Daily rate of nitrogen uptake in the above-ground plant parts of maize.

$-{ }_{--+C o u r b e s}$ enveloppes de l'intervalle de confiance au seuil de 5 p. 100 ; Confidence limits for $P=0.05$ -.. Courbe moyenne; Average curve 
de parcelles $\mathrm{N}=0$ sont de l'ordre de $35 \mathrm{~kg} / \mathrm{ha}$ (fig. 8) malgré un reliquat d'azote avant labour de $70 \mathrm{~kg} / \mathrm{ha}$ sur l'horizon $0-70 \mathrm{~cm}$. Plusieurs hypothèses peuvent expliquer cette faible contribution : lessivage important par les pluies d'avril et mai, faible minéralisation (sol à 1,1 p. 100 de matière organique), exploitation restreinte du milieu par le système racinaire des plantes de la parcelle sans azote.

\section{Phosphore (fig. 6 et 7)}

Par rapport aux 2 autres éléments majeurs, la cinétique d'absorption est très régulière et le niveau de prélèvement beaucoup plus modéré $\left(90 \mathrm{~kg} / \mathrm{ha} \mathrm{P}_{2} \mathrm{O}_{5}\right.$, dont les $2 / 3$ pour le grain). Les besoins ont tendance à augmenter lors de la phase de production maximale de matière sèche et lors de la formation et le début du grossissement du grain.

\section{Potassium (fig. 6 et 7)}

a) Besoins globaux : Les prélèvements maximums par la plante entière, au stade brunissement des soies, sont très importants : $322 \mathrm{~kg} / \mathrm{ha}$ de $\mathrm{K}_{2} \mathrm{O}$, dont $56 \mathrm{~kg}$ dans le système racinaire. Au stade ensilage, l'ensemble des parties aériennes a prélevé $260 \mathrm{~kg}$ de $\mathrm{K}_{2} \mathrm{O}$. A maturité physiologique, $40 \mathrm{~kg}$ /ha sont exportés par le grain $\left(0,350 \mathrm{~kg} \mathrm{~K} \mathrm{~K}_{2} \mathrm{O} / \mathrm{q}\right.$ de grain à $\left.15 \mathrm{p} .100\right)$.

b) Rythme d'absorption : Bien que présentant de nombreuses similitudes avec celle de $N$, la cinétique d'absorption se caractérise par un démarrage plus rapide, une plus forte intensité des prélèvements en phase active de croissance de la plante et la cessation de toute absorption après le brunissement des soies.

\section{4) Méso-éléments (fig. 6 et 7)}

a) Calcium et magnésium : Les niveaux de prélèvements totaux de $\mathrm{Ca}$ et $\mathrm{Mg}$ sont respectivement de $56 \mathrm{~kg} / \mathrm{ha} \mathrm{CaO}$ et $36 \mathrm{~kg} / \mathrm{ha} \mathrm{MgO}$. Durant tout le cycle végétatif du mais, il y a similitude du rythme d'absorption, mais les intensités sont différentes. Nettement plus élevé en période de production maximum de M.S. (14-16 feuilles), le prélèvement décroît ensuite pour cesser à la formation du grain. Par contre, la répartition de ces éléments dans les organes diffère très sensiblement : $\mathrm{Ca}$ s'accumule particulièrement dans les feuilles et plus modérément dans les tiges, $\mathrm{Mg}$ se localisant dans le grain qui contient à la récolte $3 \mathrm{p} .100 \mathrm{du}$ Ca absorbé et 49 p. $100 \mathrm{du} \mathrm{Mg}$.

b) Soufre: Bien que modestes, les besoins en $\mathrm{S} d u$ maîs $(24 \mathrm{~kg} / \mathrm{ha})$ sont également à prendre en considération. La cinétique des prélèvements relativement régulière fait apparaître 2 stades d'absorption préférentielle : 14-16 feuilles et formation des grains, 54 p. $100 \mathrm{du}$ total étant localisés dans ces derniers.

\section{Oligo-éléments (fig. 9)}

a) Zinc : Les prélèvements totaux en $\mathrm{Zn}$ sont relativement élevés : $600 \mathrm{~g} / \mathrm{ha}$, dont $37 \mathrm{p}$. 100 sont exportés par le grain. L'absorption démarre précocement, ce qui explique l'apparition des symptômes de déficience dès le stade 5 feuilles en sol carencé, et demeure soutenue jusqu'au brunissement des soies.

b) Cuivre: Les prélèvements sont de l'ordre de $90 \mathrm{~g} / \mathrm{ha}$. Le grain ne prélève que $20 \mathrm{p} .100$ et l'on constate une certaine accumulation au niveau du système racinaire.

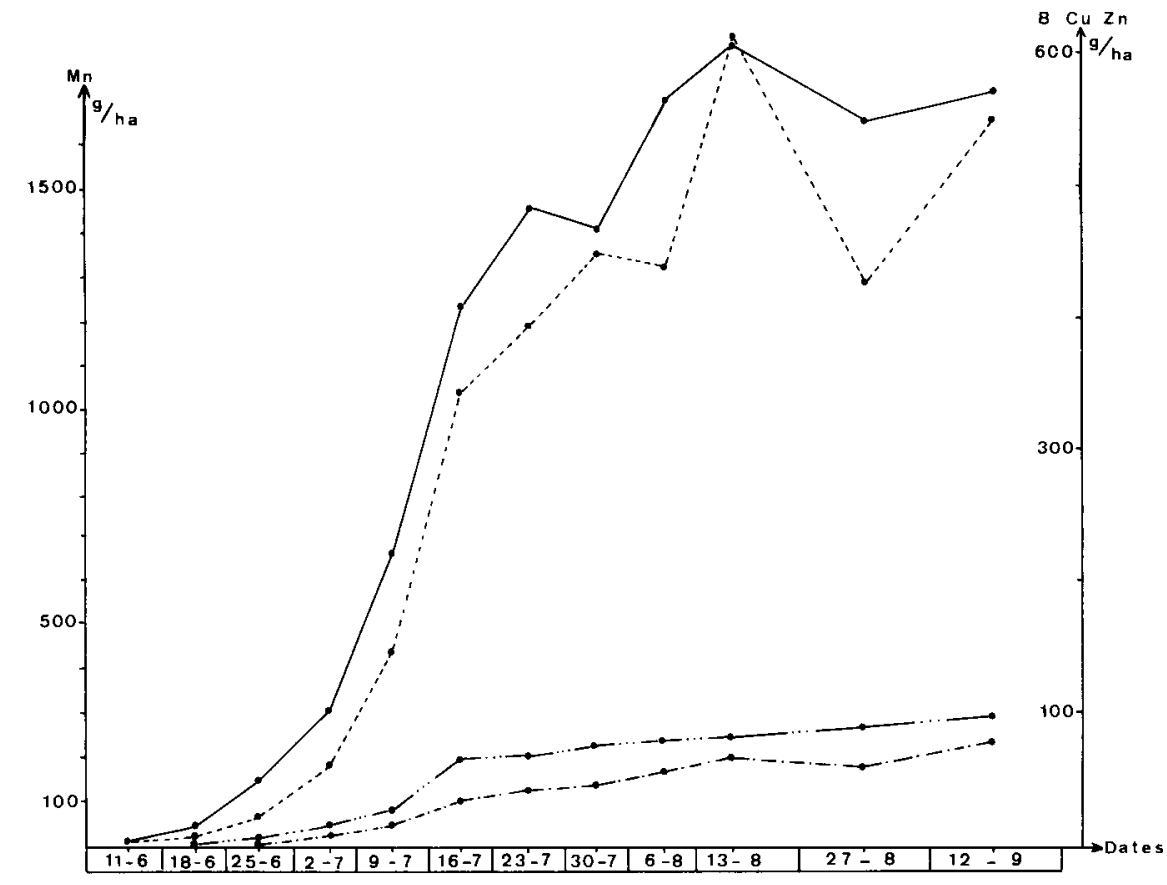

Figure 9

Rythme de prélèvement des oligo-éléments ; Micronutrient uptake by maize plants.

$\longmapsto \mathrm{Zn} ; \cdot--\cdot \mathrm{Mn} ; \cdot-\cdots \rightarrow \mathrm{Cu} ;-\cdot-\rightarrow \mathrm{B}$. 
c) Bore: Le rythme d'absorption de cet oligoélément est régulier jusqu'à maturité. Le total prélevé représente $75 \mathrm{~g} / \mathrm{ha}$, dont 50 p. 100 sont exportés par le grain.

d) Manganèse : Si les prélèvements apparaissent élevés (1 $800 \mathrm{~g} / \mathrm{ha}$ ) avec un rythme d'absorption identique à celui du zinc, les exportations par le grain sont insignifiantes (moins de 3 p. 100 du total de $\mathrm{Mn}$ absorbé).

\section{CONCLUSION}

Bien qu'issues d'un seul dispositif, les informations recueillies au cours de ce travail paraissent susceptibles d'être généralisées pour des conditions de sol et de climat voisines de celles décrites car elles ont été obtenues à partir d'un site expérimental et d'une culture que l'on peut considérer comme très homogènes et très représentatifs. Par ailleurs, elles corroborent fidèlement les résultats d'une enquête concernant le prélèvement maximum, réalisée en 1976 par la Station d'Agronomie de Bordeaux dans des cultures de maîs d'une même tardiveté, situées dans des milieux analogues.

L'élaboration, en $120 \mathrm{j}$, de $21479 \mathrm{~kg}$ de matière sèche confirme la très forte capacité photosynthétique du maïs liée à son appartenance au groupe $\mathrm{C} 4$. Cette potentialité $(180 \mathrm{~kg} / \mathrm{ha}$ de M.S. produits en moyenne chaque jour) paraît se situer nettement au-delà des valeurs $(120-130 \mathrm{~kg} / \mathrm{j}$ ) indiquées par BARLOY (1983). Malgré les conditions climatiques défavorables qui ont régné durant la phase d'installation de la culture, la somme élevée de température $\left(1977^{\circ}\right)$ relevée durant la phase expérimentale, somme très supérieure à celle observée en moyenne sur 20 ans $\left(1860^{\circ}\right)$, a certainement contribué à l'obtention d'un rendement élevé.

Cependant, on peut se demander si le déficit d'insolation relevé durant la $3^{\text {e }}$ décade de juillet (de l'ordre de 40 p. 100 par rapport à la normale) n'a pas limité la photosynthèse (source) et, par voie de conséquence, le rendement, l'efficience photosynthétique chez le maïs étant plus élevée avant la floraison (BONHOMME, 1983 ; DERIEUX, 1983).

Cette importante production de matière sèche, avec des pointes journalières atteignant près de $500 \mathrm{~kg} / \mathrm{ha}$, entraîne une mobilisation considérable d'éléments nutritifs qui doivent, en outre, se trouver dans le sol à forte concentration et être disponibles durant la période où les exigences instantanées sont très élevées. Le raisonnement de la fertilisation devra impérativement tenir compte de cette notion de forte disponibilité instantanée, au moment où il n'est pratiquement plus possible d'intervenir par des moyens conventionnels. Le problème se pose surtout pour l'azote, la méthode des bilans prévisionnels mise au point pour le blé paraissant difficilement applicable, notamment en raison de la difficulté de prédiction de la capacité de minéralisation pour la courte période correspondant aux besoins intenses du maïs. En l'état actuel des connaissances, l'apport d'azote, en quantité sensiblement équivalente au prélèvement maximum, doit être réalisé, dans la majorité des cas, juste avant le semis. Dans les sols à faible C.E.C., l'apport en localisation profonde dans l'interligne en cours de végétation constitue la technique la mieux adaptée à une meilleure efficience de cet élément et la plus apte à limiter les risques de pollution du sous-sol.

Pour les autres éléments, le problème se pose en termes différents : en effet, les sols fertilisés en tenant compte des résultats fournis par l'analyse de terre et des éléments du système de culture dans lequel s'insère le maïs (précédent cultural, maîtrise de l'eau, amendements organiques...) sont à même d'assurer une alimentation satisfaisante de la plante durant le déroulement de tout le cycle végétatif. Dans ce cas-là, le raisonnement de la fertilisation devra viser au moins à compenser par des apports annuels (éléments majeurs) ou pluriannuels (méso et oligo-éléments) les exportations prévisibles en fonction de l'objectif de rendement espéré (tabl. 4).

Reçu le 13 mars 1984. Accepté le 22 octobre 1984.

\section{REMERCIEMENTS}

Les auteurs remercient MM. J.-P. SOYER et J. TAUZIN (I.N.R.A., Station d'agronomie de Bordeaux) pour l'aide qu'ils ont bien voulu apporter à l'analyse statistique et à la représentation graphique des résultats.

TABLEAU 4

Prélèvements et exportations effectives pour une production de $115 q /$ ha de grain à 15 p. 100 d'humidité. Nutrient uptake and exportation for a maize grain yield of 11.5 t/ha at $15 \%$ moisture.

\begin{tabular}{|c|c|c|c|c|c|c|c|c|c|c|}
\hline & \multicolumn{6}{|c|}{ en $\mathrm{kg} / \mathrm{ha}$} & \multicolumn{4}{|c|}{ en $g / h a$} \\
\hline & $\mathrm{N}$ & $\mathrm{P}_{2} \mathrm{O}_{5}$ & $\mathrm{~K}_{2} \mathrm{O}$ & $\mathrm{CaO}$ & $\mathrm{MgO}$ & $\mathrm{S}$ & B & $\mathrm{Cu}$ & $\mathrm{Zn}$ & Mn \\
\hline Prélèvements maxima & 241 & 90 & 322 & 56 & 36 & 25 & 75 & 90 & 594 & 1794 \\
\hline $\begin{array}{l}\text { Exportations par : } \\
-\quad \text { parties aériennes stade ensilage } \ldots \ldots \ldots \ldots \ldots \\
- \text { grain, récolte } 29 \% \text { d'humidité } \ldots \ldots \ldots \ldots \ldots \\
- \text { récolte épi (grain }+ \text { rafle) } \ldots \ldots \ldots \ldots \ldots \ldots\end{array}$ & $\begin{array}{l}206 \\
131 \\
135\end{array}$ & $\begin{array}{l}84 \\
62 \\
62\end{array}$ & $\begin{array}{r}260 \\
40 \\
50\end{array}$ & $\begin{array}{l}50 \\
1,4 \\
1,6\end{array}$ & $\begin{array}{l}32 \\
15 \\
15\end{array}$ & $\begin{array}{l}21 \\
13 \\
15\end{array}$ & $\begin{array}{l}66 \\
41 \\
45\end{array}$ & $\begin{array}{l}62 \\
19 \\
23\end{array}$ & $\begin{array}{l}455 \\
203 \\
222\end{array}$ & $\begin{array}{r}1345 \\
48 \\
55\end{array}$ \\
\hline 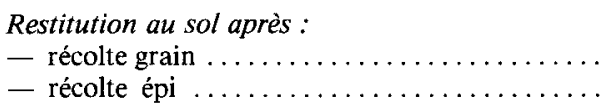 & $\begin{array}{r}100 \\
96\end{array}$ & $\begin{array}{l}21 \\
21\end{array}$ & $\begin{array}{l}259 \\
249\end{array}$ & $\begin{array}{l}52 \\
52\end{array}$ & $\begin{array}{l}19 \\
19\end{array}$ & $\begin{array}{l}13 \\
11\end{array}$ & $\begin{array}{l}57 \\
53\end{array}$ & $\begin{array}{l}70 \\
66\end{array}$ & $\begin{array}{l}312 \\
293\end{array}$ & $\begin{array}{l}1660 \\
1653\end{array}$ \\
\hline
\end{tabular}




\section{RÉFÉRENCES BIBLIOGRAPHIQUES}

Barloy J., 1983. Biologie de l'élaboration du rendement. Coll. "Physiologie du Mais ", Royan (France), 15.03.1983 (Croissance et Développement), 26 p., en cours d'impression.

Bonhomme R., 1983. Mise en place des appareils foliaire et racinaire chez le Maïs. Coll. "Physiologie du Maïs », Royan (France), 15.03.1983 (Croissance et Développement), 35 p., en cours d'impression.

Brouwer R., 1963. Some physiological aspects of the influence of growth factors in root medium on growth and dry matter production. Meded. 212 Jaarb., I.B.S., 11-30.

Cauderon A., 1980. Génétique, sélection et expansion du maiss en France depuis 30 ans. Cultivar, 133, 13-19.

Courpron C., 1974. Pertes en éléments fertilisants dans les sols sableux des Landes soumis à une fertilisation et une irrigation intensives. Ann. Agron., 25 (2-3), 467-482.

Courpron C., Tauzin J., 1971. Rythme de l'absorption des éléments minéraux par une culture de maiis irriguée, en sol sableux des Landes. C. R. Acad. Agric. Fr., 57, 479-492.

Derieux M., 1983. Sélection et adaptation. Coll. «Physiologie du Maïs », Royan (France), 17.03.1983 (Adaptation au milieu et diversité génétique), $32 \mathrm{p}$., en cours d'impression.

Girardin Ph., 1982. Essais de modélisation de la croissance et du développement du mais grain. Thèse Dr. Ing. Ecologie Végétale, Univ. Paris, Orsay, $\mathrm{n}^{\circ}$ 526, $145 \mathrm{p}$.
Hanway J. J., 1962. Corn growth and composition in relation to soil fertility. I. Growth of different plant parts and relation between leaf weight and grain yield. Agronomy J., 54, 145-148.

Hanway J. J., Russel W. A., 1969. Dry matter accumulation in corn (Zea mays) plants : comparisons among single cross hybrids. Agronomy J., 64, 947-951.

Johnson R. R., Mc Clure K. E., Johnson L. J., Klosterman E. W., Triplett C. B., 1966. Corn plant maturity. I. Changes in dry matter and protein distribution in corn plants. Agronomy J., 58, 151-153.

Juste C., Tauzin J., Dureau P., Courpron C., 1982. Exportation des éléments fertilisants par lessivage en sols sableux des Landes de Gascogne. Résultats de 8 années d'observations en cases lysimétriques. Agronomie, 2 (1), 91-98.

Mengel D. B., Barber S. A., 1974. Development and distribution of the corn root system under field conditions. Agronomy J., 66, 341344.

Morot-Gaudry J. F., Jolivet E., 1983. Assimilation photosynthétique du carbone chez le maïs. Coll. "Physiologie du mais », Royan (France), 16.03.1983 (Nutrition et Productivité), 34 p., en cours d'impression.

Sharp R. E., Davies W. J., 1979. Solute regulation and growth by roots and shoots of water-stressed maize plants. Planta, 147, 43-49. 